\title{
COMPARISON OF SOIL MODELS IN THE THERMODYNAMIC ANALYSIS OF A SUBMARINE PIPELINE BURIED IN SEABED SEDIMENTS
}

\author{
Waldemar Magda \\ Gdańsk University of Technology, Faculty of Civil and Environmental Engineering, \\ Department of Geotechnics, Geology and Marine Civil Engineering, Poland
}

\begin{abstract}
This paper deals with mathematical modelling of a seabed layer in the thermodynamic analysis of a submarine pipeline buried in seabed sediments. The existing seabed soil models: a "soil ring" and a semi-infinite soil layer are discussed in a comparative analysis of the shape factor of a surrounding soil layer. The meaning of differences in the heat transfer coefficient of a soil layer is illustrated based on a computational example of the longitudinal temperature profile of a-kilometer long crude oil pipeline buried in seabed sediments.
\end{abstract}

Keywords: submarine buried pipeline, seabed thermal models, shape coefficient, heat transfer coefficient, longitudinal temperature profile

\section{INTRODUCTION}

Heat transfer (based on convection and/or conduction phenomena), from a medium transported by a submarine pipeline (e.g., crude oil or natural gas) to a seabed sediment environment surrounding a buried submarine pipeline, takes place in a pipeline thermodynamic system consisting of several adjacent layers variable in thickness, formed by different materials/substances of different thermodynamic properties.

In general, the following component layers can be distinguished in the thermodynamic system of a partially/ fully buried submarine pipeline (Figs. 1 and 2):

a) inside film (formed by a transported medium),

b) anti-corrosion inside coating,

c) steel pipe,

d) anti-corrosion outside coating,

e) thermal insulation,

f) anti-buoyancy (weight) and protective concrete coating, g) outside film (formed by seawater flowing around a pipeline - only in the case of an exposed or partially buried submarine pipeline),

h) seabed soil medium surrounding a partially or fully buried submarine pipeline.

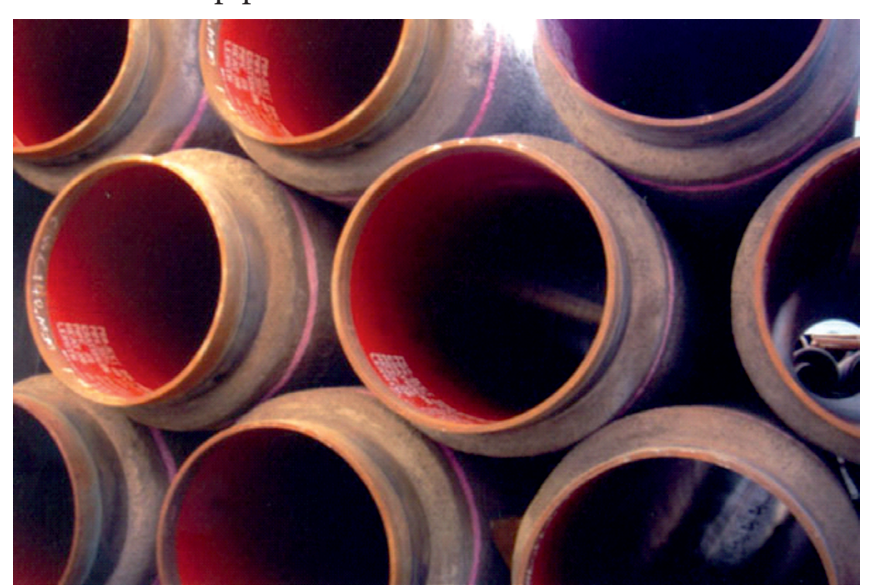

Fig. 1. Thick-walled steel pipe coated with $3 M$ Scotchkote epoxy coating EP2306 HF, and a heavy concrete coating, for $370 \mathrm{~km} \times 48 \mathrm{in}$ Dolphin (subsea) gas pipeline [10] 


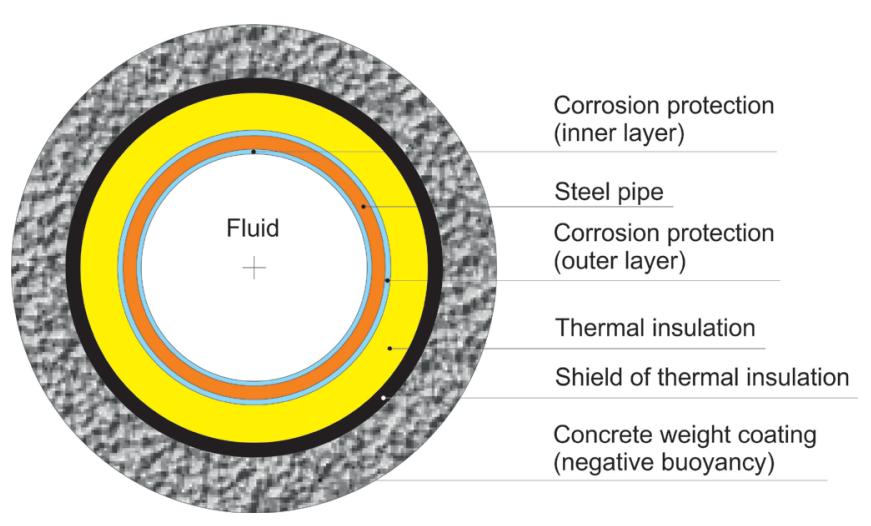

Fig. 2. Cross-section of a typical submarine pipeline structure [8]

The design procedures, established for submarine pipelines either laid on the seabed or buried in seabed sediments, require to take into account the phenomena of heat transfer through a multi-layered thermal barrier of the pipeline structure. In most cases from the list given above (i.e., from (b) to (f), and (h) under certain conditions), the component layers are combined serially and have an annular (in 2D) or a cylindrical (in 3D) shape.

The basic parameters, used in the pipeline thermodynamic analysis and characterizing the component layers, are as follows:

- thermal conductivity $\lambda[\mathrm{W} /(\mathrm{m} \cdot \mathrm{K})]$ of the material creating a component layer (a material parameter),

- shape factor $S[-]$ of a component layer (a geometric parameter),

- heat transfer coefficient $U\left[\mathrm{~W} /\left(\mathrm{m}^{2} \cdot \mathrm{K}\right)\right]$ of a component layer (a combined material-geometric parameter).

\section{SINGLE CYLINDRICAL THERMAL BARIER}

Taking into account a single cylindrical layer considered in the thermodynamic analysis, the heat transfer coefficient is given by the following equation

$$
U^{(1)}=\frac{\lambda^{(1)}}{r_{i}^{(1)} \ln \left(\frac{r_{o}^{(1)}}{r_{i}^{(1)}}\right)}
$$

where:

$U^{(1)}$ - heat transfer coefficient of a single cylindrical thermal barier $\left[\mathrm{W} /\left(\mathrm{m}^{2} \cdot \mathrm{K}\right)\right]$,

$\lambda^{(1)}$ - thermal conductivity of material forming a single thermal barier $[\mathrm{W} /(\mathrm{m} \cdot \mathrm{K})]$,

$r_{i}^{(1)}-$ inner radius of a single cylindrical thermal barrier [m], $r^{(1)}$ - outer radius of a single cylindrical thermal barrier [m].

In order to describe geometrically a cylindrical thermal barrier, the following non-dimensional shape factor can be used

$$
S^{(1)}=U^{(1)} \frac{2 \pi r_{i}^{(1)}}{\lambda^{(1)}}
$$

Comparing the last two equations, the shape factor of a single cylindrical thermal barrier takes the final practical form

$$
S^{(1)}=\frac{2 \pi}{\ln \left(\frac{r_{o}^{(1)}}{r_{i}^{(1)}}\right)}
$$

It is obvious that the shape factor of a single cylindrical thermal barrier depends only on geometric dimensions, i.e.: the inner and outer radii of the cylindrical barrier.

A surface conducting heat in a single cylindrical thermal barier is not constant, it depends on the computational radius, $r$, where $r_{i}^{(1)}<r<r_{o}^{(1)}$. Therefore, for practical purposes, one of the two characteristic surfaces of a single cylindrical thermal barrier, i.e.: either the inner or the outer, is usually assumed and defined in derivation of Eq. (1). In the above inquiry the inner surface is assumed, indicated by parameter $r_{i}^{(1)}$ appearing in the first term of the denominator of Eq. (1) and in Eq. (2).

\section{MULTI-LAYERED CYLINDRICAL THERMAL SYSTEM}

In the simplest case, heat transfer through a single thermodynamically homogeneous barrier is considered. However, when a submarine pipeline structure is concerned, it may be necessary to compute the overall heat transfer coefficient, taking into account all meaningful adjacent component layers, thus creating a multi-layered pipeline thermodynamic system.

Adopting the continuity principle for the steady-static heat transfer through the component layers of the buried pipeline thermodynamic system, the overall heat transfer coefficient (also known as the Peclet's coefficient) can be obtained from an electrical resistance analogy between heat transfer and direct current

$$
U=\frac{1}{\frac{1}{U_{f i}}+\frac{1}{U_{a i}}+\frac{1}{U_{p}}+\frac{1}{U_{a o}}+\frac{1}{U_{i}}+\frac{1}{U_{c}}+\frac{1}{U_{s}}}
$$

where:

$U$ - overall heat transfer coefficient of the multi-layered thermodynamic system of the pipeline buried in seabed sediments $\left[\mathrm{W} /\left(\mathrm{m}^{2} \cdot \mathrm{K}\right)\right]$,

$U_{f i}$ - heat transfer coefficient of the inside film (boundarylayer between the pipe and the medium transported) $\left[\mathrm{W} /\left(\mathrm{m}^{2} \cdot \mathrm{K}\right)\right]$

$U_{a i}$ - heat transfer coefficient of the inner anti-corrosion coating $\left[\mathrm{W} /\left(\mathrm{m}^{2} \cdot \mathrm{K}\right)\right]$,

$U_{p}-$ heat transfer coefficient of the steel pipe $\left[\mathrm{W} /\left(\mathrm{m}^{2} \cdot \mathrm{K}\right)\right]$, 
$U_{a o}$ - heat transfer coefficient of the outer anti-corrosion coating $\left[\mathrm{W} /\left(\mathrm{m}^{2} \cdot \mathrm{K}\right)\right]$,

$U_{i}$ - heat transfer coefficient of the thermal insulation coating $\left[\mathrm{W} /\left(\mathrm{m}^{2} \cdot \mathrm{K}\right)\right]$,

$U_{c}$ - heat transfer coefficient of the concrete weight coating $\left[\mathrm{W} /\left(\mathrm{m}^{2} \cdot \mathrm{K}\right)\right]$,

$U_{s}$ - heat transfer coefficient of the seabed soil layer $\left[\mathrm{W} /\left(\mathrm{m}^{2} \cdot \mathrm{K}\right)\right]$

The overall heat transfer coefficient (OHTC) of the pipeline is also called the $U$-value of the pipeline in subsea engineering. From the engineering practice viewpoint it is possible and recommended to simplify Eq. (4) by excluding some components appearing in the main denominator, however, not bringing any meaningful effects to the quality of computational results.

For insulated pipelines, thermal insulation coatings provide an order of magnitude larger thermal resistance than both the inside film (always existing) and the outside film (existing only in the case of totally exposed or partially buried pipelines). Therefore the effects of internal and external film coefficients $\left(U_{f i}\right.$ and $\left.U_{f o}\right)$ to the overall $U$-value of the pipeline can be practically ignored.

The internal and external anti-corrosion coatings are rather thin, made of materials which do not have significant thermal resistances. Therefore the influence of both anti-corrosion coatings can be practically omitted in Eq. (4).

The steel pipe wall-thickness is usually much smaller than the thickness of concrete coating. On the other hand the thermal conductivity of steel is much larger than the conductivity of concrete or seabed soil (see Tables 1 and 2). Consequently, due to a very small temperature drop within the steel pipe wall, the existence of the steel pipe can be also neglected in the thermodynamic analysis of the submarine pipeline.

Tab. 1. Thermal conductivities of selected materials/substances [8]

\begin{tabular}{|l|c|}
\hline \multicolumn{1}{|c|}{ Material / Substance } & $\begin{array}{c}\text { Thermal conductivity } \\
\lambda[\mathrm{W} /(\mathrm{m} \cdot \mathrm{K})]\end{array}$ \\
\hline Air & 0.026 \\
\hline Water & 0.6 \\
\hline Sand (water saturated) & $1.2 \div 2.8$ \\
\hline Aerogel (@ $\left.\mathrm{t}=0^{\circ}\right)$ & $0.012 \div 0.016$ \\
\hline Epoxy foam & 0.07 \\
\hline Polyurethane foam & $0.03 \div 0.4$ \\
\hline Polypropylene foam & $0.12 \div 0.2$ \\
\hline Syntactic foam & $0.08 \div 0.15$ \\
\hline Phase Change Material $(\mathrm{PCM})$ & $0.12 \div 0.17$ \\
\hline Concrete & $0.8 \div 1.4$ \\
\hline Steel $(0.2 \% \mathrm{C})$ & 50 \\
\hline
\end{tabular}

Tab. 2. Thermal conductivities of typical soil surrounding a pipeline [1]

\begin{tabular}{|l|c|}
\hline \multicolumn{1}{|c|}{ Type of soil } & $\begin{array}{c}\text { Thermal conductivity } \\
\lambda[\mathrm{W} /(\mathrm{m} \cdot \mathrm{K})]\end{array}$ \\
\hline Peat (wet) & 0.54 \\
\hline Sand (soaked) & $1.90 \div 2.42$ \\
\hline Clay (wet) & $1.04 \div 1.56$ \\
\hline
\end{tabular}

Thermal conductivities of concrete and seabed soil are comparable $\left(\lambda_{c} \approx \lambda\right.$, see Tables 1 and 2$)$. Therefore the conditions $\lambda \gg \lambda_{c} \approx \lambda_{s}$ means that the multi-layered thermodynamic system of the pipeline buried in seabed sediments can be simplified and approximated by a threelayer system where the thermal insulation, the concrete coating and the seabed surrounding soil are basic regulators of the heat transfer process

$$
U=\frac{1}{\frac{1}{U_{i}}+\frac{1}{U_{c}}+\frac{1}{U_{s}}}
$$

Insulation manufacturers typically use a $U$-value based on the outer diameter of an entire pipeline structure, while pipeline designers use a $U$-value based on the inner diameter [1].

The insulation and concrete coatings have an annular/ cylindrical shape, so that computation of the heat transfer coefficients, $U_{i}$ and $U_{c}$, can be performed using Eq. (1). However, as far as seabed soil sediments in the proximity of the submarine buried pipeline is concerned, the question of proper modelling of the soil area/volume arises. For the analysis of heat transfer from the pipeline structure to seabed soil sediments the following two soil models can be adopted:

- "soil ring" model (annular/cylindrical layer of soil adjacent to the pipeline structure),

- soil half-space model (semi-infinite layer of soil surrounding the pipeline structure).

The "soil ring" model is an artificial model, still assuming axisymmetrical heat transfer from the medium transported outwards. The soil half-space model reflects a real situation of the submarine pipeline buried in seabed sediments with an asymmetrical heat transfer from the pipeline structure to seabed soil sediments.

\section{SEABED SOIL MODELS FOR A TOTALLY BURIED PIPELINE}

In order to simplify the following considerations leading to a comparison of the above mentioned two seabed soil models, it is assumed that the pipeline structure is only a onecomponent (one-layer) structure, made of a steel pipe (Fig. 3). Additionally, as recommended in [1], it is assumed that the overall heat transfer coefficient is based on the inner diameter of the entire pipeline structure. It means that computations of all component heat transfer coefficients have to involve the inner diameter of the pipeline structure, represented by the inner diameter of the steel pipe, $r_{i}^{(p)}$ (see Fig. 3).

\section{“SOIL RING” MODEL}

Assuming the "soil ring" model (see Fig. 3), the heat transfer coefficient is given by the following equation (see Eq. (1)) 


$$
U_{s} \equiv U_{s 1}=\frac{\lambda_{s}}{r_{i}^{(p)} \ln \left(\frac{r_{o}^{(s)}}{r_{i}^{(s)}}\right)}
$$

where:

$U_{s}$ - heat transfer coefficient of soil $\left[\mathrm{W} /\left(\mathrm{m}^{2} \cdot \mathrm{K}\right)\right]$,

$U_{s 1}^{s}$ - heat transfer coefficient of the "soil ring" model $\left[\mathrm{W} /\left(\mathrm{m}^{2} \cdot \mathrm{K}\right)\right]$,

$\lambda$ - thermal conductivity of soil (see Table 2$)[\mathrm{W} /(\mathrm{m} \cdot \mathrm{K})]$,

$r_{0}^{(s)}$ - outer radius of the "soil ring" [m],

$r_{i}^{(s)}$ - inner radius of the "soil ring" [m],

$r_{i}^{(p)}-$ inner radius of the steel pipe $[\mathrm{m}]$.

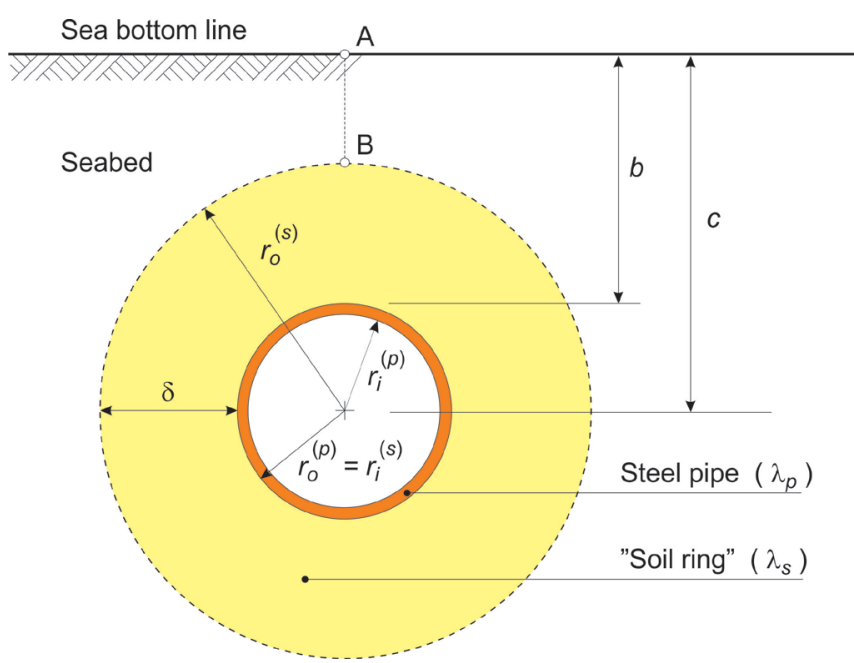

Fig. 3. The "soil ring" model assumed in the thermodynamic analysis of a submarine pipeline buried in seabed sediments [4]

According to Cranmer [4], the outer radius of the "soil ring" must be equal to a threefold value of the inner radius of the "soil ring"

$$
r_{o}^{(s)}=3 r_{i}^{(s)}
$$

It implies the following thickness of the "soil ring"

$$
\delta=r_{o}^{(s)}-r_{i}^{(s)}=2 r_{i}^{(s)}
$$

It is obvious that Eq. (6) can only be applied when the depth of burial fulfills the following geometric condition (see Fig. 3)

$$
b \geq r_{o}^{(s)}-r_{i}^{(s)}
$$

which, in conjunction with Eq. 7, takes the form

$$
b \geq 2 r_{i}^{(s)}
$$

where $b$ is the depth of burial of the submarine buried pipeline, measured vertically from the seabed bottom line to the top of the pipeline structure, [m].

After incorporating Eq. (7) into Eq. (6), the heat transfer coefficient of the soil modelled by the "soil ring" can be written as

$$
U_{s} \equiv U_{s 1}=0.91 \frac{\lambda_{s}}{r_{i}^{(p)}}
$$

whereas the shape factor of the "soil ring" is constant, irrespectively of the geometry of the system considered (see Eq. (3))

$S_{s}=U_{s} \frac{2 \pi r_{i}^{(p)}}{\lambda_{s}} \equiv S_{s 1}=2 \pi \cdot 0.91=5.72=\mathrm{const}$

where:

$S_{s} \quad$ - shape factor of a soil layer $[-]$,

$S_{s 1}$ - shape factor of the soil layer modelled by the "soil ring" $[-]$.

The idea of the "soil ring" was also used by Phetteplace [9]. Recalling the following basic relation

$$
U=\frac{1}{R}
$$

where:

$U$ - heat transfer coefficient $\left[\mathrm{W} /\left(\mathrm{m}^{2} \cdot \mathrm{K}\right)\right]$,

$R$ - heat transfer resistance $\left[\mathrm{m}^{2} \cdot \mathrm{K} / \mathrm{W}\right]$,

the equations presented in [8] can be simply reorganized in order to achieve the following relations:

- for $\frac{c}{r_{i}^{(s)}}>1$

$$
U_{s 2^{\prime}}=\frac{\lambda_{s}}{r_{i}^{(p)}\left[\ln \left(\frac{c}{r_{i}^{(s)}}\right)+\sqrt{\left.\left(\frac{c}{r_{i}^{(s)}}\right)^{2}-1\right]}\right.}
$$

- for $\frac{c}{r_{i}^{(s)}}>4$

$$
U_{s 2^{\prime \prime}}=\frac{\lambda_{s}}{r_{i}^{(p)} \ln \left(2 \frac{c}{r_{i}^{(s)}}\right)}
$$

Adequate equations for the shape factor of soil are as follows:

- for $\frac{c}{r_{i}^{(s)}}>1$

$$
S_{S} \equiv S_{s 2^{\prime}}=\frac{2 \pi}{\ln \left(\frac{c}{r_{i}^{(s)}}\right)+\sqrt{\left(\frac{c}{r_{i}^{(s)}}\right)^{2}-1}}
$$


- for $\frac{c}{r_{i}^{(s)}}>4$

$$
S \equiv S_{s 2^{\prime \prime}}=\frac{2 \pi}{\ln \left(2 \frac{c}{r_{i}^{(s)}}\right)}
$$

\section{SOIL HALF-SPACE MODEL}

Additionally to the "soil ring" model, the soil half-space model (i.e. semi-infinite soil layer) was proposed in the literature $[1,5,6]$. The half-space model is specially dedicated to the case where Eq. (6) cannot be used because of the following condition

$$
b<2 r_{i}^{(s)}
$$

For such a case, a more general model was worked out, where the heat transfer coefficient of soil layer was derived assuming the soil area in form of the half-space surrounding the pipeline structure. The soil half-space model yields the following equations $[1,5,6]$ :

$$
\begin{gathered}
U_{s} \equiv U_{s 3}=\frac{\lambda_{s}}{r_{i}^{(p)} \operatorname{arcosh}\left(\frac{c}{r_{i}^{(s)}}\right)} \\
S_{s} \equiv S_{s 3}=\frac{2 \pi}{\operatorname{arcosh}\left(\frac{c}{r_{i}^{(s)}}\right)}
\end{gathered}
$$

where:

$U_{s 3}$ - heat transfer coefficient of the soil half-space $\left[\mathrm{W} /\left(\mathrm{m}^{2} \cdot \mathrm{K}\right)\right]$,

$S_{s 3}$ - shape factor for the soil half-space [-],

$c$ - depth of burial of the submarine buried pipeline, measured vertically from the seabed bottom line to the centre of the pipeline structure cross-section, [m],

$r_{i}^{(s)}$ - inner radius of the "pipe opening" in the soil half-space $[\mathrm{m}]$,

$r_{i}^{(p)}-$ inner radius of the steel pipe $[\mathrm{m}]$.

\section{COMPARISON OF SOIL MODELS}

A graphical comparison of the shape factor of the soil layer, $S_{s}$, for the range of relative depth of burial $c / r_{i}^{(s)}=1 \div 6$ and different soil models analysed in the present paper, is shown in Fig. 4.

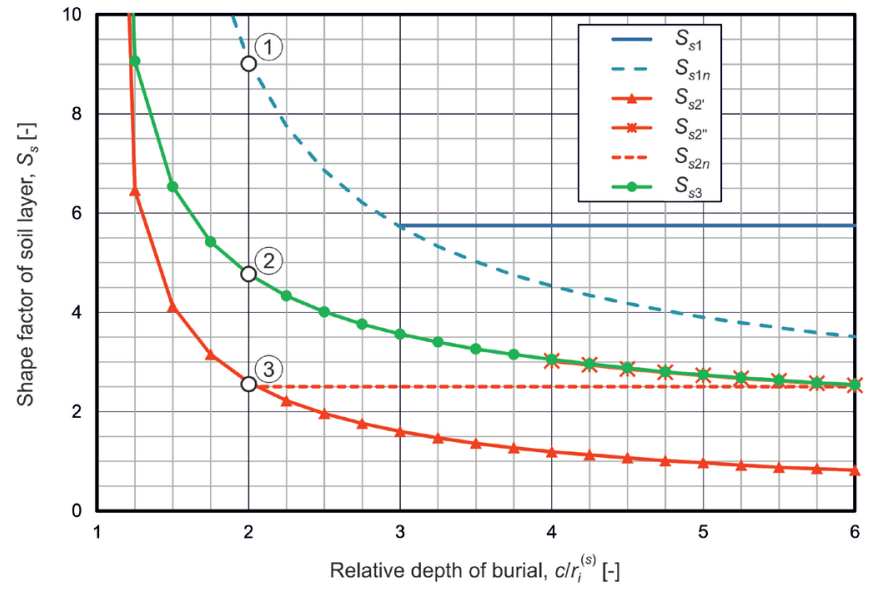

Fig. 4. Comparison of the shape factor of the soil layer surrounding the submarine pipeline structure vs. the relative depth of burial, for different seabed soil models

Based on the comparative analysis, the following findings can be drawn:

- A high convergence of curves $S_{s 3}$ and $S_{s 2^{2}}$ for $c / r_{i}^{(s)}=4 \div 6$ can be explained through the following relation

$$
\cosh x \approx \ln (2 x) \quad \text { for } x \gg 1
$$

that makes Eqs. (17) and (20) equivalent.

- Chronologically the pioneering proposal for the shape factor of the soil layer was introduced in [4]. The assumption of the "soil ring" model makes the shape factor always constant irrespectively of the system geometry, i.e.: $c$ and $r_{i}^{(s)}$ (for the "soil ring" thickness $\delta=2 r_{i}^{(s)}$ the shape factor was equal to $S_{s 1}=5.72$.

Based on the idea of the "soil ring" model, herewith a certain modification of the model is proposed, assuming the "soil ring" always tangential to the sea bottom line (point A coincides with point B, see Fig. 3), with a variable thickness depending on the depth of burial. It implies that

$$
c=b+r_{i}^{(s)}
$$

and

$$
S_{s 1 n}=\frac{2 \pi}{\ln \left(\frac{c}{r_{i}^{(s)}}\right)}
$$

Of course, this new solution proposed for the shape factor $S_{\text {s1n }}$ has only one common point with the solution $S_{s 1}$, which take place for $c=3 r_{i}^{(s)}$ (see Fig. 4).

- When the relative depth of burial, $c / r_{i}^{(s)}$, decreases from 2 to 1 , the three functions $S_{s 1 n}, S_{s 3^{\prime}}$ and $S_{s 3}$ show a strong increase of the shape factor, tending asymptotically to infinity for $c / r_{i}^{(s)} \rightarrow 1$. The increase in the shape factor 
$S_{s}$ for decreasing $c / r_{i}^{(s)}$ seems to be logical but its high rate is practically abnormal, not to show any practical application. Taking the above into account, and assuming a minimum thickness of soil cover above the pipeline structure fulfilling the condition $b \geq r_{i}^{(s)}$, a practical range of applicability of the above presented equations for the shape factor of the soil layer can be stated as $c / r_{i}^{(s)} \geq 2$.

- For the practical range of the relative depth of burial $c / r_{i}^{(s)} \geq 2$, the range of variation of $S_{s 2^{2}}$ and $S_{s 2^{\prime}}$ is reasonably small and equal to: $S_{s 2^{2}}=2.59 \div 1.19$ for $c / r_{i}^{(s)}=2 \div 4$ and $S_{s 2^{\prime \prime}}=3.02 \div 2.53$ for $c / r_{i}^{(s)}=4 \div 6$. Consequently, it seems to be fully justified to assume a practical approximation of the soil model presented in [9], assuming a constant value of the shape factor of the soil layer, i.e. $S_{s 2 \mathrm{n}}=2.5$ (see Fig. 4).

- As far as the curves of $S_{s 1 n}, S_{s 2 n}$ and $S_{s 3}$ are concerned, one can note that the solution obtained for $S_{s 3}$ creates an average solution for both extreme solutions denoted by curves $S_{s 1 n}$ and $S_{s 2 n}$.

\section{LONGITUDINAL TEMPERATURE PROFILE - COMPUTATIONAL ANALYSIS}

The meaning of the reported differences in the shape factor values for different seabed soil models will be illustrated based on a computational analysis of a temperature distribution of a medium transported along a submarine buried pipeline. The input data set consists of the following items:

$L=100 \mathrm{~km} \quad$ length of the crude oil pipeline

$D_{o}=0.8636 \mathrm{~m} \quad$ outer diameter of the steel pipe (34 in. D)

$D_{i}=0.8271 \mathrm{~m} \quad$ inner diameter of the steel pipe

$s=0.01825 \mathrm{~m} \quad$ wall thickness of the steel pipe $(0.7185 \mathrm{in}$. WT)

$Q=5000 \mathrm{~m}^{3} / \mathrm{hr} \quad$ volumetric discharge of crude oil

$S_{r}=0.85 \quad$ specific density of crude oil

$c_{p}=1.8 \mathrm{~kJ} /(\mathrm{kg} \cdot \mathrm{K})$ specific heat of crude oil at constant pressure [2]

$\lambda_{s}=2.0 \mathrm{~W} /(\mathrm{m} \cdot \mathrm{K})$ thermal conductivity of soil (sandy seabed sediments; see Table 2)

$t_{s}=4^{\circ} \mathrm{C} \quad$ ambient temperature of seabed pipeline surroundings

$t_{1}=60^{\circ} \mathrm{C} \quad$ inlet temperature of crude oil

$J_{l}=0 \mathrm{~K} / \mathrm{m} \quad$ temperature increment of the crude oil transported along the pipeline due to the Joule-Thompson effect and possible changes in a pipeline route vertical profile

Three different values of the shape factor of the soil layer, i.e.: (1) $S=S_{s 1 n}=9.06$, (2) $S=S_{s 2}=4.77$, and (3) $S=S_{s 3 n}=2.50$, were assumed in the computational analysis. These values were formerly obtained for the seabed soil models discussed previously (see Fig. 4). The highest, practically acceptable, spread in the shape factor values was assumed to happen at the smallest practical relative depth of burial, which is equal to $c / r_{i}^{(s)}=2$.
In order to simplify the computational analysis it was additionally assumed that the entire pipeline structure is formed by a steel pipe only. A possible occurrence of additional coatings, i.e.: thermal insulation, anti-corrosion, and concrete coatings, are excluded from the present analysis in order to get a better insight into functionality of the above presented pure seabed soil models. This assumption implies the relation $r_{i}^{(s)}=r_{o}^{(p)}=D_{o} / 2$. Consequently, the outer diameter of the steel pipe equal to $D_{o}=0.8636 \mathrm{~m}$ induces the following values of the heat transfer coefficient: (1) $U_{s 1}=6.5 \mathrm{~W} /\left(\mathrm{m}^{2} \cdot \mathrm{K}\right)$, (2) $U_{s 2}=3.4 \mathrm{~W} /\left(\mathrm{m}^{2} \cdot \mathrm{K}\right),(3) U_{s 3}=1.8 \mathrm{~W} /\left(\mathrm{m}^{2} \cdot \mathrm{K}\right)$, respectively to the above computed values of the shape factor of the soil layer. The computational analysis was based on a computational algorithm presented in [5, 7], and the results obtained are illustrated in Fig. 5.

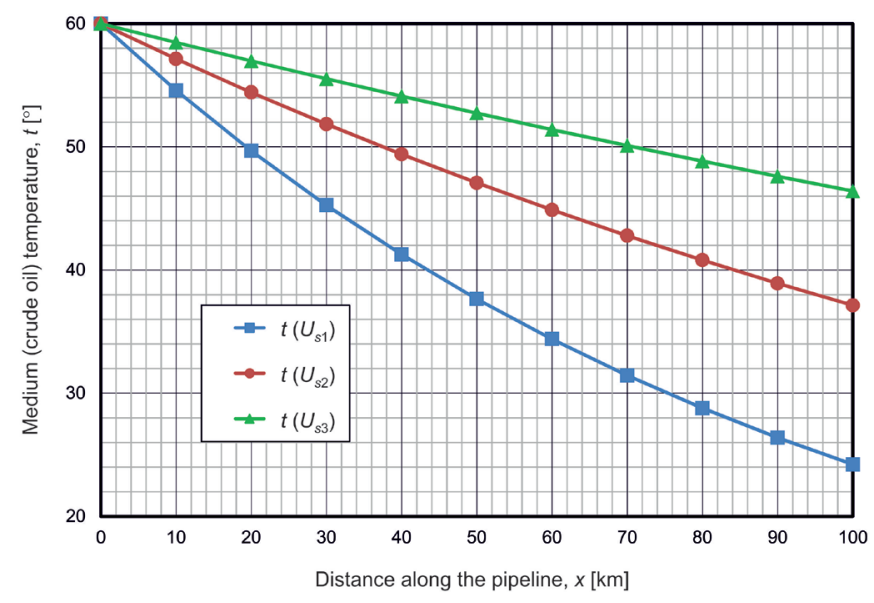

Fig. 5. The crude oil temperature distribution along the submarine buried pipeline for different values of the heat transfer coefficient of the soil layer, representing different seabed soil models

The three computational cases indicate evidently significant monotonic drops in crude oil temperature as the medium travels along the pipeline. Of course, the temperature drop effect was anticipated because the seabed soil layer (wet sandy soil) - the only additional layer considered in the pipeline thermodynamic system - acts, contrary to the steel pipe, as a relatively good thermal insulator. In general, temperature drops at the end of the pipeline depends on the pipeline length. For the $100-\mathrm{km}$ long-distance submarine buried pipeline assumed for the analysis, the computed outlet temperature (i.e. at the end-point of the pipeline) of crude oil show the temperature drop equal to: $22.7 \%, 38.1 \%$, and $59.7 \%$ of the inlet temperature set initially to $t=60^{\circ} \mathrm{C}$.

Under some special flowing conditions (temperature and pressure), the following issues: wax crystallisation, wax deposition, wax gelation and methane hydrate formation are essential in proper design procedures for submarine pipelines, especially deepwater and ultra-deepwater pipelines. Among others, a crude oil flow in the submarine pipeline can be endangered (or even terminated temporarily or permanently) when the flowing temperature drops beneath a certain critical temperature called the wax crystallisation temperature. This complex situation can be avoided only by preventing the 
pipeline thermodynamic system from excessive losses of heat energy convected and/or conduced from the flowing medium to the pipeline ambient environment. The problem becomes acute especially for long-distance submarine pipelines.

For example, assuming the crude oil to have a characteristic similar to waxycrude oils that can be found in the North Sea region (the wax crystallisation temperature ranging from $30 \div 40^{\circ} \mathrm{C}$ ), the temperature longitudinal profiles obtained for the last two values of the heat transfer coefficient ( $U_{s 2}$ and $U_{s 3}$ ) indicate the "waxy problem" that can appear in the remaining pipeline section after approximately $40 \mathrm{~km}$ and $80 \mathrm{~km}$ distances, respectively, from the pipeline inlet.

\section{CONCLUSION}

Two basic mathematical seabed soil models are discussed in the paper. Comparison of the models led to computation of the shape factor of the soil layer, giving the highest possible range thereof $\left(S_{s}=2.50 \div 9.06\right)$ obtained for the practically smallest relative depth of burial $c / r_{i}^{(s)}=2$. Consequently, the heat transfer coefficient of the soil layer was found to cover the range $U_{s}=1.80 \div 6.5 \mathrm{~W} /\left(\mathrm{m}^{2} \cdot \mathrm{K}\right)$, respectively. The smallsest values of the shape factor and the heat transfer coefficient are related with the "soil ring" model proposed in [8], whereas the largest relate to the modified Cranmer's "soil ring" solution (see Eq. (23)). The soil half space model creates the solution which averages the above mentioned extreme solutions based on the "soil ring" model of seabed soil sediments.

The meaning of the identified variability of the heat transfer coefficient of the soil layer, $U$, was illustrated performing the computational analysis of the longitudinal temperature profile in the case of the $100-\mathrm{km}$ long crude oil submarine pipeline buried in seabed sediments. The investigated variability range of the heat transfer coefficient covers different situations, e.g. relatively good thermal insulation ensured by the seabed soil layer (case 3 for $U_{s 3}=1.80 \mathrm{~W} /\left(\mathrm{m}^{2} \cdot \mathrm{K}\right.$ ), and very poor thermal insulation (case 2 for $U_{s 2}=3.40 \mathrm{~W} /\left(\mathrm{m}^{2} \cdot \mathrm{K}\right.$ ) or case 1 for $\left.U_{s 1}=6.50 \mathrm{~W} /\left(\mathrm{m}^{2} \cdot \mathrm{K}\right)\right)$ which can cause the crude oil transportation process dangerous because of the "waxy problems".

Due to a lack of suitable literature reports, at the moment it is rather difficult to judge which model of the seabed soil layer gives the best prediction of the real thermal conditions. Probably the only way to get a highly satisfactory answer to this problem is to perform detailed laboratory investigations of a temperature field around a submarine pipeline buried in seabed sediments for steady-state flowing conditions.

\section{REFERENCES}

1. Bai Y., Bai Q. (2005): Subsea Pipelines and Risers, Elsevier Science 2005.

2. Burger J., Sourieau P., Combarnous M. (1985): Thermal Methods of Oil Recovery, Éditions Technip, Paris 1985.
3. Carslaw H.S., Jaeger J.C. (1986): Conduction of Heat in Soils, Oxford University Press, second Edition, April 10, 1986.

4. Cranmer Jr. J. L. (1984): BASIC Pipeline Engineering Manual, PennWell Publishing Company, Tulsa, Oklahoma.

5. Golshan H., Zhou Z.J. (1997): Gas pipeline temperature profile predictions in discontinuous permafrost regions. Proc. of the Offshore Mechanics and Arctic Engineering (OMAE), Vol. V, Pipeline Technology, pp. 177-184.

6. King G. G. (1981): Equation predicts buried pipeline temperatures. Oil \& Gas Journal, March 16, 1981, PennWell Publishing Company, Tulsa, Oklahoma, pp. 65-72.

7. Magda W. (2005): Rurociagi podmorskie. Zasady projektowania, Wydawnictwa Naukowo-Techniczne, Warszawa 2005.

8. [8] Magda W. (2010): Nowoczesne termoizolacje rurociągów podmorskich, Inżynieria Morska i Geotechnika, No. 2/2010, pp. 244-256.

9. Phetteplace, G. (2006): What's Hot and What's Not: Defending Thermal Damage Claims, Presentation at the $97^{\text {th }}$ Annual Conference of the International District Energy Association, 11-14 June 2006, Nashville, TN.

10. http://www.ofshore-technology.com/contractors/ corrosion $/ 3 \mathrm{~m} / 3 \mathrm{~m} 2 . \mathrm{html}$.

\section{CONTACT WITH THE AUTHOR}

\author{
Waldemar Magda \\ e-mail:waldemar.magda@wilis.pg.gda.pl \\ Gdańsk University of Technology \\ Faculty of Civil and Environmental Engineering \\ Department of Geotechnics \\ Geology and Marine Civil Engineering \\ G. Narutowicza 11/12 \\ 80-233 Gdańsk \\ Poland
}

